\title{
Is the Cygnus Loop two supernova remnants?
}

\author{
B. Uyanıker ${ }^{1}$, W. Reich ${ }^{2}$, A. Yar ${ }^{1}$, R. Kothes ${ }^{1,3}$, and E. Fürst ${ }^{2}$ \\ 1 National Research Council, Herzberg Institute of Astrophysics, Dominion Radio Astrophysical Observatory, \\ PO Box 248, Penticton, British Columbia, V2A 6K3, Canada \\ e-mail: aylin.yar@.nrc.ca, roland.kothes@nrc.ca \\ 2 Max-Planck-Institut für Radioastronomie, Auf dem Hügel 69, 53121 Bonn, Germany \\ e-mail: wreich@mpifr-bonn.mpg.de, efuerst@mpifr-bonn.mpg.de \\ 3 Department of Physics and Astronomy, The University of Calgary, 2500 University Dr. NW, \\ Calgary, AB, T2N 1N4 Canada
}

Received 26 March 2002 / Accepted 26 May 2002

\begin{abstract}
The Cygnus Loop is classified as a middle-aged supernova remnant (SNR) located below the Galactic equator $\left(\ell=74^{\circ}, b=-8^{\circ} .6\right)$ and $770 \mathrm{pc}$ away from us. Its large size and little confusion with Galactic emission makes it an ideal test ground for evolutionary and structural theories of SNRs. New radio continuum mapping of the Cygnus Loop at $2695 \mathrm{MHz}$ with the Effelsberg 100-m telescope provides indications that the Cygnus Loop consists of two separate SNRs. Combining this result with data from the literature we argue that a secondary SNR exists in the south with a recently detected neutron star close to its center. Two interacting SNRs seem to be the best explanation to account for the Cygnus Loop observations at all wavelengths.
\end{abstract}

Key words. ISM: magnetic fields - supernova remnants - radio continuum - polarization

\section{Introduction}

The unusual shape of the Cygnus Loop has been the focus of interest, because this SNR, by being close to us ( $\sim 70$ pc, Minkowski 1958) and being relatively unaffected by the complex Cygnus region emission, provides most detailed information on middle-aged SNRs. Having these properties, the Cygnus Loop is in general considered to be the laboratory of SNR studies in the X-ray, optical, infrared, and radio.

New polarimetric data towards the Cygnus Loop at $2695 \mathrm{MHz}(\lambda=11 \mathrm{~cm})$ reveal that this remnant in fact consists of two separate SNRs. The analysis of these data together with available information on the rotation measure (RM), X-ray, optical, and infrared observations of the Cygnus Loop are used to constrain the characteristics and the relation of both SNRs.

\section{Radio data at $2695 \mathrm{MHz}$}

We refer to a new sensitive radio map obtained with the Effelsberg 100-m telescope at $2695 \mathrm{MHz}$ with an angular resolution of 4.3 including linear polarization (Fig. 1). The map was observed as part of a multi-frequency continuum and polarization study (Uyaniker et al., in prep.). The shape of the Cygnus Loop is circular in the north

Send offprint requests to: B. Uyanıker, e-mail: bulent.uyaniker@.nrc.ca with an extension to the south. The southern part of the remnant resembles a crescent whose western part is incomplete and is mixing with weak peripheral emission. Nevertheless, the southern crescent is brighter than most of the northern portions of the Cygnus Loop. The difference in total intensity between these two regions and spectral index variations of $\Delta \alpha \sim 0.2$ led Green (1990) to conclude that the northern part of the remnant is governed by a compression of the magnetic field whereas the southern part results most likely from shock acceleration. The morphological appearance of the total intensity emission in the north also supports the idea that the shock of the explosion compresses and deforms the magnetic field and thus the remnant carries the signature of the local magnetic field (Green 1984). In this way the partial shells of the remnant are aligned along the direction of the magnetic field.

The total intensity and polarization images (Fig. 1) reveal a new feature of the Cygnus Loop. There is a diffuse radio plateau enveloping the conventional bright radio appearance of the remnant. This plateau is the counterpart of those in the X-ray and infrared emission. It is prominently seen towards the north and north-eastern shell of the remnant. Thus with the detection of the radio plateau a general agreement in the radio, X-ray and infrared appearance of the remnant for its northern shell is obtained. This plateau was not detected in very early Effelsberg observations (Keen et al. 1973). 

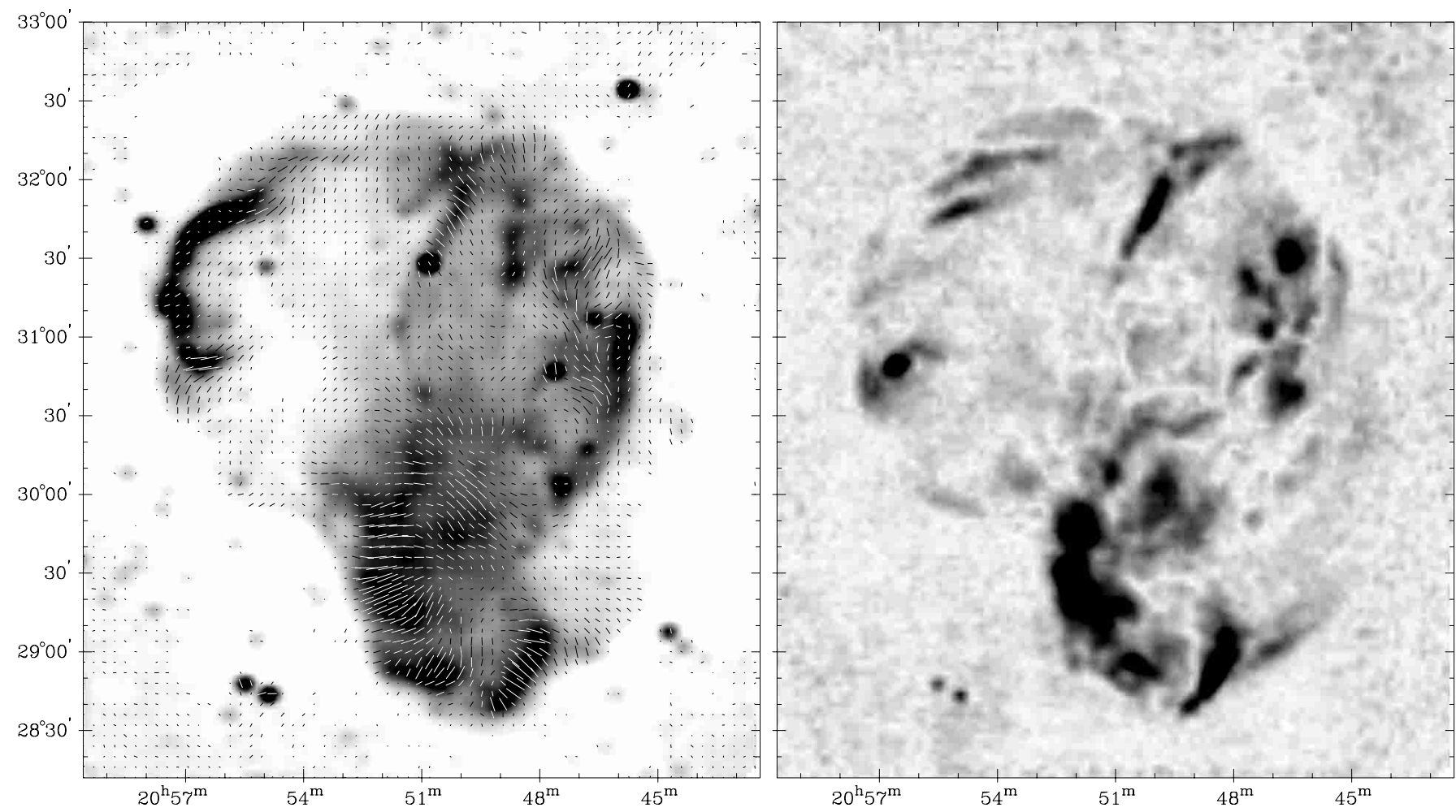

Fig. 1. Radio images of the Cygnus Loop at $2695 \mathrm{MHz}$ at a resolution of 4.3 . Both images are in the equatorial coordinate system (J2000). The total intensity map is shown on the left panel, where grayscale coding represents continuous intensity levels from $0 \mathrm{mK} T_{\mathrm{B}}$ (white) to $500 \mathrm{mK} T_{\mathrm{B}}$ (black). The overlaid bars are electric field vectors proportional to the polarized intensity. The polarized intensity image of the same region is displayed on the right hand panel. Here the grayscale coding represents levels from $0 \mathrm{mK} T_{\mathrm{B}}$ (white) to $90 \mathrm{mK} T_{\mathrm{B}}$ (black).

\section{Polarization data}

The new polarization image of the remnant shows a fair amount of polarization towards the south (up to $30 \%$ ), as well as the prominent filament almost dividing the northern shell into two symmetrical half shells. However, there is a clear distinction in the distribution of the polarized emission. The percentage polarization in the northern part is drastically lower than in the south, especially away from the polarized central filament in the north. RM calculations from the reprocessed four bands of the $1420 \mathrm{MHz}$ Dominion Radio Astrophysical Observatory (DRAO) archival interferometric maps (previously published by Leahy et al. 1997) together with the presented $2695 \mathrm{MHz}$ data (Uyanıker et al., in prep.), as well as a previous analysis by Kundu \& Becker (1972), suggest that RMs vary across the remnant between -10 to $-36 \mathrm{rad} / \mathrm{m}^{2}$. The mean is about $-20 \mathrm{rad} / \mathrm{m}^{2}$. Such a $\mathrm{RM}$ will rotate the observed polarization angle at $2695 \mathrm{MHz}$ by about $15^{\circ}$. Therefore the $2695 \mathrm{MHz}$ map traces the orientation of the magnetic field towards the remnant closely.

Consequently, the asymmetric polarization distribution between the southern and the northern part is not due to a RM gradient, but rather intrinsic. Differences in magnetic field configuration between south and north indicate the action of different mechanisms in these regions. Furthermore, while the polarized intensity in the southern part delineates a secondary circular shell corresponding to the previously called "break-out" region with magnetic field vectors aligned tangentially, the northern part displays a rather complicated appearance. We emphasize that the upper part of the polarized southern shell overlaps with the northern shell, but results from a shock originating in the south. This contradicts the previous interpretation that a shock from the northern area breaks out into the southern region. Indeed, Tenorio-Tagle et al. (1985) proposed that the northern shell of the Cygnus Loop is the breakout of a SNR in the south. However, their model has difficulties to explain the high X-ray emission from the northern shell. Furthermore, this model needs ambient material of higher density in the south - which is neither observed in the infrared (Arendt et al. 1992), in H I (DeNoyer 1975) or CO emission (Scoville et al. 1977).

We conclude from the above arguments that neither a southern nor a northern breakout explains the available observations. There is strong evidence for two separate remnants rather than a single object.

The presence of the anomalous X-ray source AX J2049.6+2939 detected by the ASCA satellite (Miyata et al. 2001) at the center of the southern remnant provides further proof that this part is independent from the large 

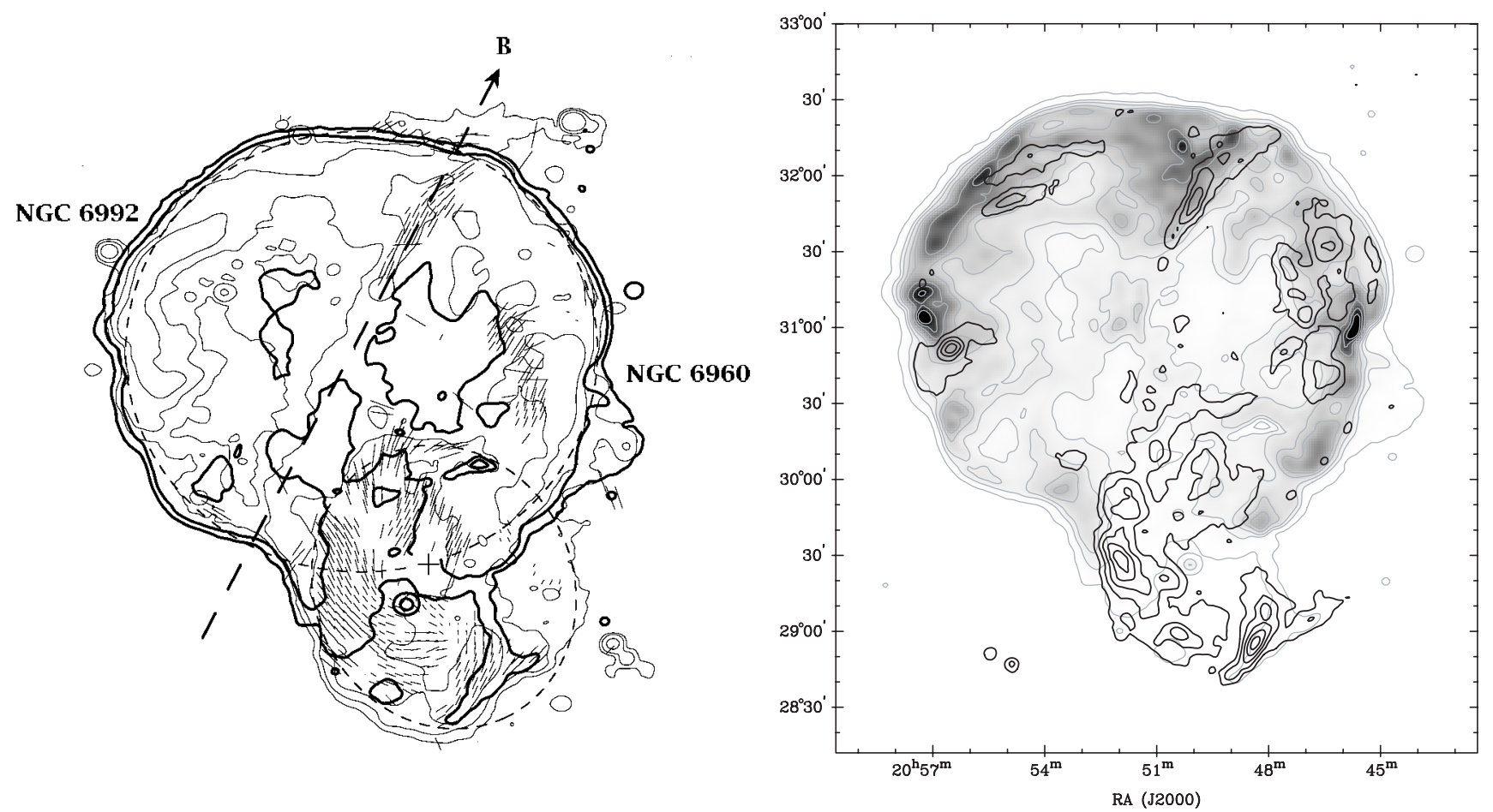

Fig. 2. Left panel: Sketch of the Cygnus Loop showing radio emission (thin lines) and $0.25 \mathrm{keV}$ X-ray emission from ROSAT data (thick lines, convolved with a Gaussian beam of $3^{\prime}$ ). The positions and extents of the two SNRs are represented by dashed ellipses. The magnetic field vectors, obtained by using the polarization image given in Fig. 1 and corrected for Faraday rotation are overlaid as bars. Note that pixels with low signal to noise ratio are omitted and the length of the vectors are proportional to RM. The vectors of strong point sources are screened to avoid confusion. The direction of the large-scale magnetic field in this area of the Milky Way is shown by a dashed straight line, which passes through the polarized central filament in the north. The neutron star in the southern part is indicated by a plus sign. The two prominent optical filaments, NGC 6992 and NGC 6960 are also labeled. Right panel: X-ray emission from the Cygnus Loop at $0.25 \mathrm{keV}$ convolved with a $3^{\prime}$ beam. The X-ray intensities range from from 0.002 counts s$^{-1}$ arcmin $^{-2}$ to 0.25 counts s$^{-1} \operatorname{arcmin}^{-2}$. Overlaid thick contours represent polarized intensity levels starting at $40 \mathrm{mK} T_{\mathrm{B}}$ running in steps of $40 \mathrm{mK} T_{\mathrm{B}}$.

northern shell. The X-ray spectrum of this source results in a photon index of $-2.1 \pm 0.1$. Although no pulsed X-ray or radio emission was detected towards this object, Miyata et al. (2001) conclude that this object is a stellar remnant, probably a neutron star. Any relationship between this $\mathrm{X}$-ray source and the southern part strengthens the idea that the Cygnus Loop consists of two SNRs.

\section{Optical, X-ray and infrared data}

Optical images (Levenson et al. 1998) reveal differences between the northern and the southern part of the Cygnus Loop. For example, $\mathrm{H} \alpha$ and O III regions are more extended in the north than in the south as a consequence of differences in the interaction with the surrounding gas.

The X-ray (Ku et al. 1984; Levenson et al. 1998) and infrared emission (Braun \& Strom 1986) of the northern part is consistent with a limb-brightened shell of hot gas, while the southern source is almost invisible. This difference was explained as a sign for different mechanisms acting in the north and the south of the Cygnus Loop (Green 1990).
We notice that at all wavelengths there are different emission characteristics in the northern part compared to the southern region.

Aschenbach \& Leahy (1999) have interpreted the southern part of the remnant as a breakout into a lower density medium. Their main reason was the smooth change of the X-ray emission from the northern to the southern area. However, a smooth transition may also result from two SNRs seen in superposition. In Fig. 2 (right panel) we display the soft X-ray image with overlaid polarization intensity contours showing a relation of minimum X-ray emission and maximum polarized emission in the area where the southern shell superimposes with the northern one. In addition there are two regions from $\sim \alpha, \delta=20^{\mathrm{h}} 54^{\mathrm{m}}, 30^{\circ}$ to $\sim \alpha, \delta=20^{\mathrm{h}} 53^{\mathrm{m}}, 29^{\circ} 30^{\prime}$ at the eastern and at $\sim \alpha, \delta=20^{\mathrm{h}} 48^{\mathrm{m}}, 29^{\circ} 45^{\prime}$ at the western side, where X-rays are enhanced and also the periphery of both shells superimpose. These findings strongly suggest that both SNR shells are not just seen in superposition, but are in physical interaction. Enhanced soft X-rays might emerge from the deceleration of the shock waves due to the collision of the SNRs. On the other hand it is difficult to understand the minimum X-ray emission in the 
overlapping region of both SNRs. We note in that context that Williams et al. (1997), who studied the colliding SNRs DEM L316 in the Large Magellanic Cloud, did not find X-ray emission in the region of overlap of both SNRs. We conclude that details of the conditions in the interacting regions are not well constrained and need a more detailed investigation

According to the interaction scenario the SNR in the north exploded first and created a complete circular, shelltype SNR. A second SN event took place at the position of AX J2049.6+2939, not far away from the already expanding shell of the first explosion. Shortly after the second explosion the shock waves of the SNRs collided. The younger southern SNR had a higher energy density than the northern one, swept-up the shell of the first remnant and bent it inward. This way the pronounced upper polarized shell of the southern SNR was created. The collision of the two shells also created the X-ray extensions encompassing the polarized emission (see Fig. 2). Comparable distances of the SNRs, however, cannot be directly utilized to estimate the quantitative ages of these SNRs by simply comparing their sizes, since at least the northern remnant is suspected to be expanding in a pre-existing cavity (Charles et al. 1985; Levenson et al. 1997).

\section{Discussion}

We have presented new radio continuum measurements of the Cygnus Loop including linear polarization and analyzed the differences between its southern and northern part. These differences are rather obvious by inspecting the polarized intensity image. We also report on the detection of a weak radio plateau positionally coinciding with those observed in the infrared and in X-rays. The following reasons led us to conclude that two SNRs make up the Cygnus Loop and are in interaction.

1) The radio morphology of the Cygnus Loop differs in the south and north. There is an elliptical shell in the north, including a radio plateau corresponding to $\mathrm{X}$ ray and infrared emission. A bright southern extension is present.

2) The distribution of polarization intensity differs completely in the south and north. RMs are not high enough to depolarize the polarized emission at $2695 \mathrm{MHz}$ significantly, indicating an intrinsic difference in the magnetic field configuration.

3) The tangential magnetic field structure indicates a regular magnetic field configuration in the southern part, but disturbed in the region of overlap, whereas it is more chaotic in the north.

4) The radio spectral index changes across the Cygnus Loop indicating a steeper spectrum (less compression) for the southern part, which in turn implies a different acceleration mechanism.
5) The northern part has different characteristics of the optical filaments compared with the southern region.

6) There is weak or no X-ray and infrared emission in the south in contrast to the strong radio appearance.

7) X-ray enhancements seen at the superposition of both shell's periphery and decreased X-ray emission in the overlap regions clearly indicate an interaction of both SNRs.

8) Finally, there is evidence for the existence of a stellar remnant, probably a neutron star, almost exactly at the center of the southern shell.

The northern remnant is centered at $\sim(\alpha, \delta)=$ $\left(20^{\mathrm{h}} 51^{\mathrm{m}} 36,31^{\circ} 3^{\prime}\right)$ and its extent is about $3.0 \times 2.6$. The southern remnant, whose extent is about $1.4 \times 1$. 8 , is centered at $\sim(\alpha, \delta)=\left(20^{\mathrm{h}} 49{ }^{\mathrm{m}} 56,29^{\circ} 33^{\prime}\right)$. Thus we designate these remnants as G74.3-8.4 and G72.9-9.0 according to their Galactic coordinates, respectively. The results from a comparative investigation of new radio data at other frequencies including a spectral index analysis and a detailed description of the polarization characteristics will be given elsewhere.

Acknowledgements. We thank Nancy Levenson for providing us with the $0.25 \mathrm{keV}$ ROSAT mosaic and Tom Landecker for critical reading of the manuscript and useful discussions.

\section{References}

Aschenbach, B., \& Leahy, D. A. 1999, A\&A, 341, 602

Arendt, R. G., Dwek, E., \& Leisawitz, D. 1992, ApJ, 400, 562

Braun, R., \& Strom, R. G. 1986, A\&AS, 63, 345

Charles, P. A., Kahn, S. M., \& McKee, C. F. 1985, ApJ, 295, 456

DeNoyer, L. K. 1975, ApJ, 196, 479

Green, D. A. 1984, MNRAS, 211, 433

Green, D. A. 1990, AJ, 100, 1927

Keen, N. J., Wilson, W. E., Haslam, C. G. T., Graham, D. A., \& Tomasson, P. 1973, A\&A, 28, 197

Ku, W. H.-M., Kahn, S. M., Pisarski, R., \& Long, K. S. 1984, ApJ, 278, 615

Kundu, M. R., \& Becker, R. H. 1972, AJ, 77, 459

Leahy, D. A., Roger, R. S., Ballantyne, D. 1997, AJ, 114, 2081

Levenson, N. A., Graham, J. R., Aschenbach. B., et al. 1997, ApJ, 484, 304

Levenson, N. A., Graham, J. R., Keller, L. D., \& Richter, M. J. 1998, ApJS, 118, 541

Minkowski, R. 1958, Rev. Mod. Phys., 30, 1048

Miyata, E., Ohta, K., Torii, K., et al. 2001, ApJ, 550, 1023

Scoville, N. Z., Irvine, W. M., Wannier, P. G., \& Predmore, C. R. 1977, ApJ, 216, 320

Tenorio-Tagle, G., Rozyczka, M., \& Yorke, H. W. 1985, A\&A, 148,52

Williams, R. M., Chu, Y.-H., Dickel, J. R., et al. 1997, ApJ, 480,618 